\title{
Longitudinal qualitative evaluation of pharmacist integration into the urgent care setting
}

This article was published in the following Dove Press journal: Integrated Pharmacy Research and Practice

\section{David J Wright' \\ Richard J Adams' \\ Jeanette Blacklock' \\ Sarah A Corlett ${ }^{2}$ \\ Rebecca Harmston ${ }^{3}$ \\ Margaret McWilliams ${ }^{3}$ \\ Stephen-Andrew Whyte ${ }^{4}$ \\ Gail Fleming ${ }^{4}$}

'School of Pharmacy, University of East Anglia, Norwich, UK; ${ }^{2}$ Medway School of Pharmacy, Universities of Greenwich and Kent at Medway, Chatham, UK; ${ }^{3}$ Patient and public involvement member of the research team, University of East Anglia, Norwich, UK; ${ }^{4}$ Health Education England, London, UK
Correspondence: David J Wright School of Pharmacy, University of East Anglia, Norwich Research Park, Norwich, Norfolk, NR4 7TJ, UK

Tel +441603592042

Email d.j.wright@uea.ac.uk
Purpose: To describe the most effective model for managing, educating, and training pharmacist advanced clinical practitioners (ACPs) in the urgent care center (UCC) setting, role evolution and how to measure their effectiveness.

Participants and methods: Ethical approval was obtained to perform a qualitative longitudinal cohort study in three sites, with three pharmacists in each trained as ACPs from 2016 to 2017. ACP role, location, management, mentorship, and supervision were locally determined. ACPs attended focus groups (FGs) at 1 and 3 months (sites 1-3), 6 and 12 months (site 1 only), and the UCC staff were interviewed once with a topic guide regarding training, integration, role, and impact. Verbatim transcriptions were analyzed thematically.

Results: Eight ACP FGs and 24 stakeholder interviews produced major themes of communication, management, education and training, role, and outcomes. Effective education, training, and integration required communication of role to address concerns regarding salary differentials, supportive management structure, and multi-professional learning. ACPs reported that the model of workplace training, experiential learning, and university-based education was appropriate. Training was better located in the minor injuries and general practitioner areas. Recommended measures of effectiveness included patient satisfaction and workload transfer.

Conclusion: The education and training model was appropriate. Communication and management require careful consideration to ensure effective integration and role development. Pharmacists were better located initially in the minor illness rather than major trauma areas. Quality of patient experience resulting from the new role was important in addition to reassurance that the role represented a positive contribution to workload.

Keywords: advanced clinical practitioner, accident and emergency, education, training, focus groups, interviews

\section{Introduction}

Between 2004 and 2014, a 35\% increase in demand in accident and emergency (A\&E) hospital attendances was recorded in the UK. ${ }^{1,2}$ This understandably impacted on the ability of hospitals to meet the UK government target that "within 4 hours of arrival, $95 \%$ of patients must be either admitted, transferred, or discharged". ${ }^{2}$ Between October and December 2015, the proportion of patients spending longer than 4 hours in $A \& E$ reached its highest level in over a decade in nine out of ten hospitals. ${ }^{2}$ In 2016, it was concluded that most A\&E departments in the UK were working at a very high level of activity, and "there is a limit to the workload staff can undertake in the absence 
of additional staff without it having negative consequences on morale, recruitment and retention, performance, and/or patient safety". ${ }^{2}$ With shortages of both doctors and nurses throughout the National Health Service (NHS), the use of other health care professionals, such as pharmacists, to address the problem was identified as a potential solution. ${ }^{3}$

Urgent care centers (UCCs) were introduced by the NHS England in $2015^{4}$ to enable patients to receive timely review by a primary care clinician and many were consequently sited within A\&E departments to reduce pressure within this area.

As early as 1974, a pharmacist was reported as joining an A\&E department in the USA ${ }^{5}$ with $87 \%$ of the physicians agreeing that the pharmacist is capable of offering primary care to certain patients once the diagnosis has been made by a physician. Additionally, 95\% physicians felt the role of the pharmacist was transferrable to other emergency room facilities. In the UK in 2005, the Queen's Medical Centre, Nottingham, ${ }^{6}$ reported that a pharmacist was working within an A\&E department undertaking medication history taking, providing advice to medical staff, attending ward rounds, supporting risk management, and providing staff training. A 2007 study in Rochester, USA, ${ }^{7}$ stated that despite the potential impact of pharmacists in A\&E, very few programs existed in which pharmacists undertake this role. In response to a survey, $99 \%$ of medical and nursing respondents in departments with a pharmacist ( $82 \%$ return rate) felt the A\&E pharmacist improved quality of care, $96 \%$ believed them to be an integral part of the team, and 93\% reported consulting the pharmacist at least a few times during their last five shifts. While there was willingness by physicians and nurses to accept a pharmacist within the A\&E team, the role described within the USA was primarily limited to medication history taking with minimal other direct patient care. ${ }^{8,9}$ While another study recognized the role of the pharmacist in A\&E in error interception, ${ }^{10}$ none have reported on pharmacists undertaking roles and responsibilities that are more akin to those of A\&E nurses and doctors.

In 2003, a study in a London-based A\&E department ${ }^{11}$ estimated that $8 \%$ of A\&E admissions could have been managed by the patient's community pharmacist, suggesting that pharmacists without any additional training can make useful contribution to workload. It was subsequently recommended that pharmacists in the UK should manage patients with minor ailments in A\&E. ${ }^{12}$ An analysis of 18,613 A\&E attendances across 49 sites in 2015 found that, with additional training, $27.9 \%$ of cases were believed to be manageable by a pharmacist prescriber. ${ }^{13}$ In 2014, the Royal Pharmaceutical Society (RPS), based on a pilot conducted in one region within England, ${ }^{14}$ proposed the incorporation of pharmacists into $A \& E$ in an enhanced role alongside other roles such as advanced practice nurses and physician associates. ${ }^{15}$

The Health Education England (HEE) ${ }^{16}$ subsequently supported the RPS recommendations but stated that work was required to develop the education and training model and this was dependent on the scope of practice and future role expectations. To answer these questions, HEE recruited three hospitals as pilot sites to integrate pharmacists into the urgent care setting in order to undertake clinical roles. Funding was provided for pharmacist training costs, and it was agreed that they would all need to be trained as advanced clinical practitioners (ACPs) through higher education institutes (HEIs).

ACPs are health care professionals from a wide range of backgrounds (nursing, pharmacy, paramedics, and occupational therapy). Trained to master's level, ACPs develop the knowledge and skills to enable them to take on expanding roles and scope of practice of caring for patients. ${ }^{17}$ The driver for ACP role formation was to enhance capacity and capability in multi-professional teams by supporting existing and more established roles. ${ }^{17}$ The ACP program selected for the trainee pharmacists within the pilot included modules on independent prescribing (IP), physiology, advanced patient assessment, clinical reasoning and applied physiology, clinical leadership, and emergency medicine. Completion resulted in the award of a Postgraduate Diploma in Advanced Clinical Practice.

The pilot enabled researchers to monitor the role as it evolved and to answer questions regarding how best to capture its impact. It will only be possible to answer questions regarding actual impact and value once the pharmacists were fully integrated and trained and therefore had sufficient autonomy to enable independent provision of patient care.

The aim of this study was therefore to identify and describe the most effective model for managing, educating, and training pharmacist ACPs in the UCC setting, describe how the role evolves during the training period, and identify how best to measure its effectiveness.

\section{Participants and methods Project setup and management}

The University of East Anglia (UEA) Faculty of Medicine and Health Sciences Ethics Committee and the National Health Research Authority provided approval for the project. The HEE funded an independent qualitative evaluation by the University of East Anglia.

The project management team met on a 3 monthly basis and consisted of four members of the research team, a representative from each site, and two patient and public 
involvement (PPI) members. RA (male, senior hospital pharmacist with $\mathrm{PhD}$ ), SC (female, senior lecturer with hospital pharmacy experience and $\mathrm{PhD}$ ), and JB (female, non-pharmacist senior research associate) are all formally trained in qualitative research methods. Focus groups (FGs) were undertaken by RA, SC, or JB, with one as lead and the second as moderator. Interviews were undertaken by SC or RA alone. JB and the patient and PPI members of the management committee (both non-pharmacist females) were used to ensure that data interpretation by RA and SC was not unduly biased by their previous experiences and personal perspectives. No relationship existed between the University and research sites or researchers and individuals within each site prior to study commencement. Participants had no previous knowledge of RA and JB. SC had taught two of the interviewees as undergraduates more than 3 years before the start of this project. None of the researchers had any previous interest or expertise in the research subject.

The HEE initiated a project whereby three A\&E departments in the South East England would each employ between one and five pharmacists within their UCCs. The pharmacists, while working in the departments as trainee pharmacist ACPs, were funded to undertake advanced clinical practice training ${ }^{18}$ and independent pharmacist prescriber training ${ }^{19}$ at local HEIs. In addition, clinical supervision and training were provided by medical practitioners and senior ACPs within the A\&E department. ${ }^{20}$ Pharmacy departments working in close collaboration with each UCC managed the project locally. The location for the ACPs, their induction, the development of their role, local management, and mentoring were all determined locally.

Grounded theory underpinned the methodological approach of the study.

\section{Project timing}

Start times for the project were staggered across sites to reflect when each UCC was ready to recruit and initiate training. The management committee communicated lessons learned from implementation within the first site to the other two sites. Time of taking up post for all ACPs in a department was time zero for that site. Due to sites 2 and 3 only starting trainees half way through the 12-month study period, data could only be collected for 5 months for site 2 and 4 months for site 3 .

\section{Participant recruitment}

Designated gatekeepers at each of the three trusts forwarded invitations, on behalf of UEA, to all trainee pharmacist ACPs and non-pharmacy staff to attend FGs or interview requests. Researchers contacted all participants who expressed an interest 3 to 4 days prior to the event to optimize attendance. Participants provided written, informed consent prior to commencement of all FGs or interviews.

\section{Sampling}

All trainee pharmacist ACPs participated in the evaluation for the purposes of the FGs. The management committee agreed that a group discussion with the three individuals at each site was preferable to individual interviews as this was likely to provide richer data.

It was not practically possible to sample interviewees purposively due to limited amount of time available to individuals who were concurrently working in A\&E. Furthermore, the researchers visited the site for one day only at each stage and did not have the flexibility to return. Consequently, the method of sampling was by convenience. Availability of individuals willing to consent to participate on the designated research days determined sample size.

\section{Setting}

Interviews and FGs took place within rooms, which were suitably comfortable and private, within each of the trusts. No person other than participants, RA, JB, or SC, was present at any $\mathrm{FG}$ or interview.

\section{Data collection}

Trainee pharmacist ACPs undertook FGs at months 1, 3, 6, and 12. UCC non-pharmacist staff undertook interviews approximately 3 months after the service was set up. The rationale for holding interviews with the A\&E non-pharmacist staff was that it was impractical to organize group meetings.

The planned duration of each FG was 1 hour. Researchers did not curtail FGs that exceeded the hour. Interviews lasted approximately 15-20 minutes with the time again defined by the interviewee. Due to the short nature of the study, repeat interviews were not undertaken.

The research management team developed indicative topic guides in line with the project objectives (Table 1). The researchers did not pilot the topic guides. All FGs and interviews were recorded using a digital recorder and transcribed verbatim by an experienced transcriber. The researchers did not take field notes. Interviewees or FG participants did not receive transcripts for comment or correction.

During the week prior to the FG, trainee pharmacist ACPs completed an activity diary to record the approximate 
Table I Topic guides for focus groups and interviews

\begin{tabular}{ll}
\hline $\begin{array}{l}\text { Trainee pharmacist ACPs } \\
\text { focus group topic guide }\end{array}$ & $\begin{array}{l}\text { A\&E non-pharmacist staff } \\
\text { interviews topic guide }\end{array}$ \\
\hline - Description of activities and & - Opinions on the ACP role \\
roles over preceding period & - Perceived contribution to patient \\
- Perceived contribution to & care and impact \\
A\&E and how this could be & - Impact of ACPs on A\&E staff \\
captured & - ACP training needs and priorities \\
- Views on integration into & - How the training should be \\
- department & delivered \\
- Opinions on training to date & - Additional thoughts \\
- Adentified training needs & \\
training and development & \\
- Additional thoughts & \\
\hline
\end{tabular}

Abbreviations: ACP, advanced clinical practitioner; A\&E, accident and emergency.

number of hours spent on each given activity. Completion of this enabled the research team to track changes in role as the 12 months progressed. It also encouraged the trainee pharmacist ACPs to reflect on their role and inform their responses within the FGs.

\section{Data analysis}

Transcripts of interviews and FGs were analyzed thematically. We initially undertook the evaluation of the development of the trainee pharmacist ACPs at each UCC independently and then combined the data from all three sites for the final analysis. Minor themes arising from individual sites were not included in the results as these were only locally relevant.

The research team did not identify themes in advance. RA read transcripts as frequently as required to ensure true familiarity or immersion in the data. Development of key ideas and recurrent themes resulted from highlighting of relevant phrases and words. RA derived themes from frequently represented and commonly occurring issues. Issues identified as occurring only once or infrequently but deemed important were also included as themes. Conversion of transcribed text into tables enabled creation of a framework. We did not use bespoke software during this process. The end of this stage produced a detailed record of the data, labeled into manageable chunks.

The navigation panel in Microsoft 2010 supported the process of merging themes. The process occasionally resulted in theme deletion if the initial designation of a theme was inappropriate. RA designated themes as significant if they had an impact on the training and education of the trainee pharmacist ACPs. Major themes generally resulted, naturally, from an accumulation of evidence, words, or phrases.

JB and SC read the transcripts and coding to check that the themes truly represented themes and that there were no omissions. Different descriptors used for the same themes were either renamed or changed. The study management group reviewed the results of the analysis and provided their views on the outcomes. A sample of FGs and interviews were independently reviewed by patient and public members of the management group to validate interpretation of the data. Participants did not provide feedback on the findings.

The thematic framework did not include numerical codes, as the number of themes was manageably small. RA assigned sample text to themes. JB and SA checked the process through reading the sample texts and the association with themes.

When presenting quotes in results, the aim was to demonstrate and explain evidence. We removed idioms and nonrelevant phrases from quotes presented in the results but did not correct grammar. To ensure anonymity, the interviewees from the three sites were coded as A, O, and J and numbered sequentially.

Due to differences in how ACPs presented and recorded data regarding activities undertaken the week prior to each qualitative data collection episode, their diaries were analyzed thematically to identify changes in role and activities as they occurred.

\section{Results}

Site 1 started in October 2016, with sites 2 and 3 starting in June 2017 and August 2017, respectively.

Four FGs were held with three ACPs at site 1 and two FGs at months 1 and 3 with two or three trainee pharmacist ACPs at the other two sites. One set of stakeholder interviews was held at each of the three sites involving A\&E non-pharmacist staff. Demographics of FG and interview attendees are summarized in Table 2. No one dropped out or refused to participate when they presented themselves for interview. The number approached by the gatekeepers and refusing to participate was not recorded.

Each FG took approximately 1 hour and the one-to-one interviews lasted approximately 30 minutes.

Five themes resulted from the combined analysis:

- Management

- Communication

- Education and training

- Role definition

- Outcomes 
Table 2 Summary of participant demographics

\begin{tabular}{|c|c|c|c|c|c|c|}
\hline \multirow[t]{2}{*}{ Site } & \multicolumn{2}{|c|}{ Site I (n) } & \multicolumn{2}{|c|}{ Site 2 (n) } & \multicolumn{2}{|c|}{ Site 3 (n) } \\
\hline & Male & Female & Male & Female & Male & Female \\
\hline & \multicolumn{6}{|c|}{ Focus groups } \\
\hline \multirow[t]{2}{*}{ Trainee pharmacist advanced clinical practitioners } & I & 2 & 0 & 3 & 2 & I \\
\hline & \multicolumn{6}{|c|}{ Interviews } \\
\hline Consultant & 2 & 0 & 2 & 1 & $\mathrm{I}$ & 0 \\
\hline Senior medical practitioner & $\mathrm{I}$ & 0 & $\mathrm{I}$ & I & 2 & 0 \\
\hline Senior nurse & 0 & 0 & 2 & $\mathrm{I}$ & 0 & 0 \\
\hline Nurse practitioner & $\mathrm{I}$ & 0 & 0 & 1 & 0 & 0 \\
\hline Non-pharmacist advanced clinical practitioner & $\mathrm{I}$ & 0 & 0 & 0 & 0 & 2 \\
\hline Emergency nurse practitioner & I & I & 0 & 0 & 0 & 0 \\
\hline Manager & 0 & I & 0 & 0 & 0 & 0 \\
\hline Non-pharmacist advanced clinical practitioner trainee & I & I & 0 & 0 & 0 & 0 \\
\hline
\end{tabular}

\section{Management}

Trainee pharmacist ACPs recognized the importance of selecting individuals with the most appropriate attributes and these were self-reliance, a proactive nature, and resilience; recommending that management included these within any future selection procedures. It was believed that newly qualified pharmacists may be unsuited to the role.

"that shows in confidence, whereas someone else who's reasonably newly qualified I think or still studying at the moment that shows as well, so they'll get there but it's just I think they're..." A5

Early on in the process at site 1 trainees reported requesting more local management to support them on a day-to-day basis with lack of initial access to allocated mentors identified as a barrier to development. The mentors who were identified recognized that it required extra time but knowing that this would improve workload at a later stage was an enabler.

"So it slows me down in terms of service delivery, but its also very empowering in terms of saying 'I'm going to produce 3 or 4 people who in 18 months' time the NHS is going to benefit from so that's the reward"” O6

The trainee pharmacist ACPs also recognized that they were initially a drain on other people's time rather than an assistance due to the teaching time required.

Inter-professional training sessions were identified as an effective management strategy to improve integration into the multidisciplinary team.

"like 'weekly teaching sessions' for example and that integrates all the ENPs the ANP trainees ......." O2

Pharmacist drug knowledge was reported to have assisted integration with nurses who were also trained as prescribers. "...also when it comes to the prescribing...some of the nurses I've sat in with... when they came to prescribing 'oh we've got a pharmacist sitting here, perfect person' ..." A3

Trainee pharmacist ACPs at site 1 displayed a resistance to working shift patterns, while managers of $A \& E$ recognized that such a change would enhance team working and ability to receive information at handover. A senior nurse manager recognized that in order to ensure full integration, overall management should sit within A\&E but that pharmacy should retain some influence.

\section{Communication}

Communication of the role to possible applicants was identified as requiring modification.

“...I think they needed to be clearer about what their agenda was....and also what they really wanted from us and where." J1

The trainee pharmacist ACPs received salaries, which were higher than other professional disciplines and created an additional barrier to integration, which could have been prevented by better communication regarding the rationale for the difference from the outset.

"...to be really honest I think we were quite angry that you would take two people who have never put a hand on a patient, you paid them at $8 \mathrm{a} . . .$. . to come in for people that are working as band 7 , who are doing the job to train ..." J9

A key issue with integration was that A\&E staff had not been informed that the trainee pharmacist ACPs were undertaking the training in a supernumerary manner and were funded via an external grant and this was perceived as creating tensions with other staff who were not able to access the training. By 
the time site three started, this problem had been resolved through better communication.

“...[name] was quite proactive in actually telling everybody that we've got these three pharmacists who are going to come in so we were expected... and everybody was expecting that we're going to be learning and what you're going to be doing, so it wasn't that difficult for us. It was an easy smooth in terms of integration..." A1

Similarly, trainee pharmacist ACPs stated that the amount of training required and what would be available should also have been communicated to applicants. Trainee pharmacist ACPs reported that A\&E staff had been informed about them, but that due to the numbers, staff involved were not always aware of why they were there.

“... nearly every single person is, 'are you a student phar-

macist have you worked on the wards before?" J2

The lack of awareness prompted the trainee pharmacist ACPs to undertake proactive action, with one of them offering to provide a short presentation to the regular consultants' meeting to inform them about the ACP program.

\section{Education and training}

The induction process was identified as requiring more careful consideration regarding baseline skills, structure, and duration.

“...I think because maybe the pilot sites were not too informed of what we were going to be doing, they haven't really thought about the baseline skills that we probably need ... but as a Trust or as an induction, I don't think these things have been thought out ..." A3

Involving A\&E staff in the creation of the induction program was seen as positive.

“...we've [A\&E staff] been very involved in creating an induction program we've had that autonomy which I'm really grateful for ..." J10

There were recommendations that commencement of trainee pharmacist ACPs in post and undertaking the university course should be a shorter period.

“...I'd probably say maybe not have such a long period. I think like have the induction that we've had and then start the course..." J2

ACPs and colleagues observed that, on commencement, pharmacists lack skills, which have been taught and then practiced by nurses at the same stage in training as ACPs. This included anatomy, examination skills, use of equipment, venipuncture, and some consultation skills. Specific note was made of the lack of ability of pharmacists to write concisely within medical notes.

“..and you're able to you know in three or four words kind of describe what you've just done. Instead of writing a whole paragraph that nobody really wants to kind of delve into..." $\mathrm{O} 3$

There was however approval of some elements of the trainee's consultation skills.

“...they're very good with talking to the patients explaining what's going on.” J7

A lack of awareness of "red flags" identified within the trainees resulted in the provision of a booklet at one site which was received positively.

“...but the coordinator she did give us a whole booklet

of maybe red flags to look at different conditions. Which helped quite a bit." $\mathrm{O} 2$

Site 1 had not appreciated that the effort required to deliver the training and support for the ACPs in house. The IP course was also recognized to be more demanding than expected and therefore the ACPs who had undertaken IP training prior to the ACP course were advantaged. The ACP course was judged intensive and hard work and a greater input than had been expected at recruitment.

“...coming into this course, I completely misjudged it; I thought IP would just be a walk ... I've come to learn that it's not and this course is not a joke it's serious, it's intense stuff.." A1

An experienced doctor working in A\&E stated that the trainee pharmacist ACPs may struggle to obtain all the required knowledge in the time available. The general practitioner (GP) expanded this with further observations that the expectations on the trainee pharmacist ACPs were great and that it would require very intensive training to achieve competence within 18 months.

"...we need to make sure that they have the requisite knowledge and skills to do that and giving them the 18 months. It has to be intense otherwise it had to be a kind of extended ...but I'm not sure that 18 months is adequate for someone..." A7 
The trainee pharmacist ACPs appreciated starting the HEI courses.

"...its got that structure as well and I think for this course we have to work for this programme altogether. It will not make sense for us to work outside the course and do our own thing... It works. The course itself it's structured in a way that it will facilitate you to practice what you've just learnt knowledge wise, within practice ..." A1

It was recognized that HEI training cannot be solely relied upon when dealing with skills, such as examination and diagnosis, which needed to be supplemented with workplace training.

"...now that is different in a classroom to how it is in real life and the more of that you do in real life if you're supported the better you will become. But I think it's a bit too early to say really I think they've only done cardiac exam and respiratory exam so there's quite a lot left.” J6

Ensuring structure to the workplace training was seen as necessary. Difficulties were identified by coordinating training with examination and diagnosis at university and then practicing those skills in the workplace due to availability of suitable patients, and therefore, training needed to be structured alongside the taught courses.

“...so I think that it (workplace learning) has to be matched with the work they're doing at the [University]" J10

The IP course, while being useful, was described as generic in delivery and, therefore, not focused solely on pharmacist needs such as hands on skills, whereas pharmacology skills already displayed by the pharmacists are still taught because of nurse requirements.

The variety of health care professionals working in the department and, therefore, potentially available to assist with training was stated to be a benefit.

“...I think everyone brings specialist skills yes which are important for you to learn. I think it's all part of the varied workforce that they have in .... is beneficial and that's what actually helps you as a pharmacist. Because each individual brings expertise from different areas of the medical field which is imperative towards your training." A2

Without knowing what the final role would look like, it was recognized that it was difficult to develop a holistic training program.

"I would imagine that their training needs would as I said before be similar along the lines of what the training needs of our junior doctors .. I'm not $100 \%$ sure where the end point of it is. I mean what the service what the aim is what service they're going to provide at the end if that makes any sense" J11

\section{Role}

Both trainee pharmacist ACPs and other A\&E staff recognized that pharmacists in these roles are not suited to training and subsequently working in major trauma areas, whereas minor injuries and GP areas presented the greatest opportunities. The consultant in the site $1 \mathrm{GP}$ area took the initiative to move the ACPs into that environment after realizing that their first 3 months had been difficult.

".. if I hadn't forced these guys would be languishing in the main $A \& E$ department trying to upskill themselves with complex patients getting lost and they really hated it at the beginning." $\mathrm{O} 8$

Use was made of the trainee pharmacist ACPs' medicines knowledge early on in their program. However, this was at a low level initially but appears to have increased with time and was valued by A\&E staff. Recognition was made of the ability of ACPs as pharmacists to provide help and advice about the use of medicines to both staff and patients.

“...for patients, particularly elderly patients who have lots of medications looking at medication reviews. Thinking about causes and side effects for patients and toxicity so that's a huge benefit because it is a source of expertise." J10

An initial skepticism to the inclusion of trainee pharmacist ACPs in the department was reported but this changed with time.

"....I think we were a bit sceptical to start off with... knowing that we were going to get three new pharmacists but actually I think it's working really well.” A5

There was recognition that central to the role trainees had to learn how to take a complete history.

"...the main thing was to learn how to become you know experts in examining patients how to take complete histories so that was another that was a big thing for us ...challenge as a pharmacist, we don't normally do that so yes we've learnt how to take rigorous histories...yes, so that patient will not be able to tell whether I am just a pharmacist compared to a history taken by a GP..." A1

A need to identify the relevant regulatory body and revalidation process to utilize upon the qualification of the trainee pharmacist ACPs as ACPs was reported. 
“....who's going to be your competency body who's going

to be your registration body and what's the revalidation process? So I think that needs to be thought about a bit more clearly..." O6

At 1 year, there was recognition that further planning was required for future roles and that a mixture of GP areas in A\&E and multidisciplinary clinics or ward rounds would be a useful and rewarding role. A senior doctor also expressed the view that the future role was not clear.

“...my immediate thought is I'm not sure where they're heading so is it to be able to come up with a differential diagnosis cos it seems like that's what's meant to happen I'm not completely sure..." J6

A senior doctor expressed the view that he did not know the end point for the project, who would sign off the trainee pharmacist ACPs and what qualification they would gain.

\section{Outcomes}

Initially trainee pharmacist ACPs and A\&E staff reported no benefit to the running of $A \& E$ and discussed negative effects due to the time required to teach and supervise them.

"...I suspect that within the next 6 months they will actually be contributing to the service because they will be able to deliver and see patients more independently without reference to me..." O6

By the time the trainee pharmacist ACPs had been working in A\&E for 1 year, they were feeling more competent and confident, which reflected in their activity. There was clear recognition of the need for trainee pharmacist ACPs to be competent in their role.

"so, I guess it can only be good if obviously if they're competent if they're qualified and they know what to look for and what to advise..." J12

A junior charge nurse noted that the trainee pharmacist ACPs provide benefit by reducing the load of work on other staff, and by seeing patients there may be greater patient satisfaction, as patients' perception is that they have been seen earlier in their 4-hour wait.

“...they help lower down my waiting time ...because they start pulling patients. If you pull the patients up patients get seen. I know they're presenting to somebody else so in terms of patient perception. They're being seen by somebody so rather than they think thinking they're waiting for four hours

... so that makes my job easier..." J12
Support was demonstrated with the acceptance that currently there are insufficient numbers of trained health care professionals in A\&E to meet the needs of patients.

GPs working in the department expressed support for the inclusion of pharmacist ACPs due to the shortage of resources. Support was also provided due to experience with pharmacists in the community and their medicines knowledge.

"...so if is much more resource is available we're happy

to use it...I've got no problems because pharmacists are already helping in the community..." A6

In relation to benefit to $A \& E$, a junior doctor gave the opinion that there would be definite improvements in patient care if the pharmacist ACPs were focused on medication, due to their knowledge base. The A\&E staff confirmed that the trainee pharmacist ACPs' medication-related skills were appreciated.

"...finally we have someone we can go to and ask for medication queries and yeah they actually enjoyed our presence there ..." A3

A senior doctor identified that measurement of any benefit which may be provided by pharmacist ACPs should not be attempted for a year, and then to utilize a mixture of quantitative and qualitative measures.

“...I would look at things like their activities. So a bit of quantitative feedback in terms of just trying to see how many patients they are seeing and you know and what their sort of decisions are ... some other quantitative feedback can be .. how many cannulations bloods other skills like suturing or doing x-rays ..."J10

Measures of effectiveness were identified as patient satisfaction, number of patients seen with supervisor evaluations, numbers of admissions avoided, numbers of medicinesrelated interventions, adherence to national targets, and staff retention.

\section{Trainee diaries}

At site 1, activities within the first month largely focused around history taking and role orientation. At 3 months, trainees reported undertaking examinations and discussing patients with consultants. By 6 months, they reported using their pharmaceutical knowledge more frequently by providing medicines-related advice, performing medication review, practicing prescribing, and creating management plans. At 12 months, ACPs reported undertaking diagnosis and there was a great focus on clinical decision making and patient referral. 
Trainee pharmacist ACPs at sites 2 and 3 followed a similar trajectory to that at site 1 . Site 2 reported using their pharmaceutical knowledge more frequently and undertaking examinations after 1 month. Trainees at site 3 also reported undertaking patient examination after 1 month and were ordering investigations and undertaking clinical decision making at 3 months.

\section{Discussion}

This is the first paper to describe the integration of pharmacists as ACPs into UCCs with an expectation that they undertake a similar caseload to that of medical practitioners and other ACPs working within the area. The research suggests that it is feasible for pharmacists to be educated and trained to work within the area. Additionally, they can assume some of the workload while providing the benefit of immediate access to their pharmaceutical expertise.

Although minor ailments and primary care-focused workload were more appropriate for initial education and training, there were identified knowledge gaps that still required addressing. This could be achieved either prior to commencement or early on within the induction and should include writing in medical notes, anatomy, examination skills, identification of red flags, venipuncture, and use of relevant diagnostic equipment.

The model of workplace training, consisting of teaching sessions with local practitioners, university courses encompassing IP, and advanced care practitioner training provided an appropriate backbone. However, for the education and training to be fully effective and efficient, significant local planning with respect to management, communication, and education and training is required.

There were a number of strengths associated with this evaluation. First, it was independent of both the employers and the HEE who initiated the project. The longitudinal design with regular data collection enabled identification of themes in a timely manner and the researchers to monitor trainee pharmacist ACP development. The research team reduced the potential for bias through utilization of and adherence to the topic guides combined with open, nonjudgmental questions. Similarly, the use of a non-pharmacist senior research associate and patient and public involvement members minimized bias within the interpretation process. The collation of information from both trainees and all members of the UCC team, who worked in collaboration with them, enabled triangulation of evidence thereby enhancing its validity. The use of the activity diary provided additional data, which was not captured qualitatively, supported triangulation of evidence, and helped to inform responses provided by trainees during the FGs.

The original plan was to use FGs for the health care professionals, as this would enable the identification of consensus and dissonance. Similarly, we would envisage the capture of richer data due to initiation of trains of thoughts, which may not have occurred at an individual level. Conversely, the plan for multi-professional FGs may have resulted in unbalanced representation of views due to the differences in perceived power existing between different professional groups. Due to the use of convenience sampling and pragmatic approach to the number of interviews undertaken, it is unlikely that researchers achieved data saturation. The funding for the project was for 12 months, which resulted in incomplete data sets for two sites and this was largely due to delays in site initiation.

The iterative approach, with each trust recruiting at different times, enabled lessons learned at earlier trusts to be incorporated, thus resulting in improvements to the trainee pharmacist ACP experience. From a purist perspective, using data obtained from site 1 to inform practice in the other sites may not adhere to usual expectations for research of this nature. However, the evaluation was pragmatic, and with a management committee consisting of representatives from the three sites, it was not possible or appropriate to blind them to the main messages as they arose.

The fact that this was an initial pilot designed to explore the proposal, may partly explain the identification of management as a strong theme. External funding allowed the trainee pharmacist ACPs to be supernumerary, which may not reflect reality if the funding was derived internally. Initiating the project from within the A\&E department itself may have prevented some problems but potentially created others regarding professional support and guidance.

The processes of recruitment, selection, and induction for this role, however, do require particular attention as there is a need to be clear as to expectations with respect to the role communicated both in the job advert and during the interview process. A number of the characteristics required for the role were identified that could be considered central to most roles within the health care system. However, there was clear agreement that for pharmacists to be "transferred into" and "trained for" an ACP role within a UCC, they needed to have a number of years of prior experience.

The working hours for the trainees also required careful consideration as the trainees at one of the sites were employed within the traditional nine to five work schedule, which did not align with the shift patterns of other staff. This 
was a barrier to effective communication on hand over and integration into the team. The results suggest the importance of balancing the benefits of trainee pharmacist ACPs working at the same time as their clinical supervisor against the advantages of better team integration.

The results also show that clear lines of responsibility need defining from the outset, with individual mentors and managers identified who are enthusiastic about the role and recognize the future benefit to the department. Predictable knowledge gaps were identified within the trainee pharmacists and these need to be addressed either prior to commencement of role or during the induction in order to improve their confidence and reception when starting.

Communicating the purpose of the post, structure of the training, and rationale was seen as necessary for maximizing acceptance and understanding from day one. Careful design of the induction process, with the involvement of a range of practitioners, could ensure increased awareness and understanding on both sides. For similar reasons, the UCC staff recommended involvement of the A\&E staff in the design of the pharmacist's induction within one of the trusts.

Generally, the trainee pharmacist ACPs reported positively on the university courses, with some concerns voiced regarding them being "more tailored to nurse education". This probably reflects the nature of the individual training program accessed by the pilot site trainees and not ACP training more broadly. The workplace training, although effective, particularly if undertaken in a multi-professional manner, required more careful consideration. Alignment of workplace training with the university education was possible as the trainees were supernumerary and able to identify patients to put into practice their new skills. We would suggest that the organization of local training, which largely consisted of planning for the location of trainees, would benefit from identification of learning outcomes and setting time points for their achievement. These should align with the university course timetable. Responsibility for supporting development and ensuring that the ACPs met their learning outcomes would be the responsibility of the allocated education supervisor.

Concerns regarding initial role when training completed and career trajectory were voiced. Similarly, the results suggest that the role of regulators and professional organizations in recognizing the qualification and revalidation process requires consideration. The pharmacists were best suited for location within the primary care element of A\&E. Pharmacist ACPs migrated to this area in all three sites. This however may not reflect their final location and part of their career trajectory may be to migrate into the "majors" area of A\&E.
Time will see whether this occurs and a further evaluation study would be required.

While initial skepticism to the role was identified and artificial barriers created due to a lack of awareness of role and funding source, there was widespread acceptance of the individuals in all sites at the end of the evaluation. It would be reasonable to assume that pharmacist ACPs will make a significant contribution to local workload. Most methods suggested for capturing the effect of the role related to patient satisfaction, describing the process, and assessing clinical appropriateness/effectiveness.

With the large amount of supervision provided then, it is likely to be reasonable to assume that clinical outcomes from pharmacist ACP assessments would be no different to others. Pharmacist ACPs do however cost more than many of their counterparts and have significant initial training costs. While this could be offset by their ability to offer their medicines expertise to this setting, more formal evaluation of the role with respect to actual patient outcomes and costs would help policy makers to decide whether to continue this initiative. In 2017, the Royal College of Emergency Medicine recommended that pharmacists as ACPs be included in the future workforce training strategy, ${ }^{21}$ for which they have developed a recommended curriculum and assessment strategy. ${ }^{22}$

\section{Conclusion}

To retrain pharmacists to work as part of a multi-professional team of ACPs and provide their additional pharmaceutical expertise, the qualitative results suggest that it is important to identify individuals with the right attributes. The learning curve is steep, the environment is both busy and unpredictable, and therefore having an experienced individual who is proactive, organized, and resilient in nature is important. Furthermore, the amount of work required to both the learner and department should not be underrepresented.

Management of trainees should sit within the A\&E department with professional links to pharmacy retained. Management should agree working hours prior to interview and communicate them to prospective candidates. Flexibility within this may benefit the department, patients, and trainees. Mentors for trainee pharmacist ACPs should be are aware of the potential benefits of the role and willing to undertake the additional work required.

Before the trainee pharmacists start in role, management should communicate who and what they are to all the UCC staff. This is particularly important if trainee pharmacist ACP salaries are higher than that of other individuals undertaking a similar role but without the pharmaceutical expertise. The 
trainees are best located in the minor illness area initially. An induction process should be carefully considered with respect to line management, activities to be undertaken, and when and which competencies should be achieved. Using staff working within the department to design the induction was recommended, as this improved awareness and acceptance of the new role. Finally, it is important to address known deficits in pharmacist knowledge and skills, such as writing in medical records, anatomy, venipuncture, and identification of red flags, either prior to or early on within the induction process.

The education model of training as an ACP and independent prescriber through HEIs provided structure to the training and was believed to be appropriate, if combined with workplace training.

Patient satisfaction combined with a number of process measures such as number of patients seen, readmission rate, and medicines-related problems addressed was an appropriate method for capturing the effect of pharmacist ACPs.

Acceptance of pharmacists within UCC by other professions was widely identified, as was the recognition that after 12 months, they were making a positive contribution to workload. The accessibility of pharmaceutical knowledge was an added benefit. Once trained within this area, staff acknowledged that ACPs might be able to make a wider contribution, particularly in primary care.

\section{Acknowledgments}

The project was funded by HEE. The authors would like to thank all the trainee pharmacists and staff working within the urgent care settings for their time to engage with the research and provide feedback on the process as it evolved.

\section{Disclosure}

The authors report no conflicts of interest in this work.

\section{References}

1. NHS England. A\&E Attendances and Emergency Admissions February 2016 Monthly Report [updated April 14, 2016]. Available from: https:// www.england.nhs.uk/statistics/statistical-work-areas/ae-waiting-timesand-activity/. Accessed July 4, 2018.

2. The King's Fund. What's going on in A\&E? The Key Questions Answered. 2016. Available from: https://www.kingsfund.org.uk/projects/urgent-emergency-care/urgent-and-emergency-care-mythbusters. Accessed July 4, 2018.
3. Aiello M, Terry D, Selopal N, Huynh C, Hughes E. Examining the emerging roles for pharmacists as part of the urgent, acute and emergency care workforce. Clin Pharm. 2017;9(2).

4. NHS England. Transforming Urgent and Emergency Care Services in England. 2015. Available from: https://www.england.nhs.uk/wpcontent/uploads/2015/06/trans-uec.pdf. Accessed July 4, 2018.

5. Elenbaas R, Waeckerle J, McNabney W. The clinical pharmacist in emergency medicine. Am J Hosp Pharm. 1977;34(8):843-846.

6. Foreshaw G. A career as . . a an A\&E department pharmacist. Hosp Pharm. 2005;12:61-64.

7. Fairbanks RJ, Hildebrand JM, Kolstee KE, Schneider SM, Shah MN. Medical and nursing staff highly value clinical pharmacists in the emergency department. Emerg Med J. 2007;24(10):716-718.

8. Hayes B, Donovan J, Smith B, Hartman C. Pharmacist-conducted medication reconciliation in an emergency department. Am J Health Syst Pharm. 2007;64(16):1720-1723.

9. Carter M, Allin D, Scott L, Grauer D. Pharmacist-acquired medication histories in a university hospital emergency department. Am J Health Syst Pharm. 2008;63(24):2500-2503.

10. Patanwala AE, Sanders AB, Thomas MC, et al. A prospective, multicenter study of pharmacist activities resulting in medication error interception in the emergency department. Ann Emerg Med. 2012;59(5):369-373.

11. Bednall R, McRobbie D, Duncan J, Williams D. Identification of patients attending accident and emergency who may be suitable for treatment by a pharmacist. Fam Prac. 2003;20(1):54-57.

12. Clarke C. Pharmacists in A\&E. Hosp Pharm Eur. 2014;76.

13. Hughes E, Terry D, Huynh C, et al. Future enhanced clinical role of pharmacists in emergency departments in England: multi-site observational evaluation. Int J Clin Pharm. 2017;39(4):960-968.

14. Terry D. Pharmacists are being trained to work as prescribers in A\&E, conference hears. Pharm J. 2014;292(7804):357.

15. Royal Pharmaceutical Society England. Improving Urgent and Emergency care through Better Use of Pharmacists. 2014. Available from: https:// www.rpharms.com/making-a-difference/projects-and-campaigns/ improving-urgent-and-emergency-care. Accessed July 4, 2018.

16. Health Education England. Clinical Pharmacists Could Have a Positive Impact on Patient Care in A\&E [updated April 28, 2016]. Available from: https://www.hee.nhs.uk/news-blogs-events/hee-news/clinicalpharmacists-could-have-positive-impact-patient-care-ae. Accessed July 4, 2018.

17. Ferreri SP, Greco AJ, Michaels NM, et al. Implementation of a pharmacogenomics service in a community pharmacy. $J$ Am Pharm Assoc. 2014;54(2):172-180.

18. Health Education England. Advanced Clinical Practice. 2017 Available from: https://www.hee.nhs.uk/our-work/developing-our-workforce/ advanced-clinical-practice. Accessed July 4, 2018.

19. General Pharaceutical Council. Pharmacist Independent Prescriber. 2018 Available from: https:/www.pharmacyregulation.org/education/ pharmacist-independent-prescriber. Accessed July 4, 2018.

20. NHS England. Integrating Pharmacy into Urgent Care. Available from: https://www.england.nhs.uk/commissioning/primary-care/pharmacy/ urgent-care/. Accessed July 4, 2018.

21. Health Education England, NHS England, NHS Improvement, Royal College of Emergency Medicine. Securing the Future Workforce for Emergency Departments in England. 2017 Available from: https:// improvement.nhs.uk/documents/1826/Emergency_department_workforce_plan_-_111017_Final.3.pdf. Accessed July 4, 2018.

22. The Royal College of Emergency Medicine. Emergency Medicine: Advanced Clinical Practitioner Curriculum and Assessment. 2017. Available from: http://www.rcem.ac.uk/docs/Training/EM\%20ACP\%20 curriculum\%20V2\%20Final.pdf. Accessed July 4, 2018. 
Integrated Pharmacy Research and Practice

Dovepress

\section{Publish your work in this journal}

Integrated Pharmacy Research and Practice is an international, peer-reviewed, open access, online journal, publishing original research, reports, reviews and commentaries on all areas of academic and professional pharmacy practice. This journal aims to represent the academic output of pharmacists and pharmacy practice with particular focus on integrated care. All papers are carefully

peer reviewed to ensure the highest standards as well as ensuring that we are informing and stimulating pharmaceutical professionals. The manuscript management system is completely online and includes a very quick and fair peer-review system, which is all easy to use. Visit http://www.dovepress.com/ testimonials.php to read real quotes from published authors.

Submit your manuscript here: http://www.dovepress.com/integrated-pharmacy-research-and-practice-journal 